\title{
Vortex breakdown in a water-spout flow
}

\author{
Miguel A. Herrada, ${ }^{1}$ Vladimir N. Shtern, ${ }^{2}$ and José María López-Herrera ${ }^{1}$ \\ ${ }^{1}$ E.S.I., Universidad de Sevilla, Camino de los Descubrimientos s/n, 41092 Sevilla, Spain \\ ${ }^{2}$ Shtern Research and Consulting, Houston, Texas 77096, USA
}

(Received 5 June 2013; accepted 30 August 2013; published online 20 September 2013)

The numerical study of the steady axisymmetric air-water flow in a vertical sealed cylinder, driven by the rotating top disk, describes topological transformations as the rotation intensifies. The air meridional flow (AMF) and swirl induce meridional motions of opposite directions in water. For slow (fast) rotation, the effect of AMF (swirl) dominates. For very fast rotation, large-scale regions of clockwise meridional circulation in air and water are separated by a thin layer of anticlockwise circulation adjacent to the interface in water. This pattern develops for other fluids as well. Physical reasoning behind the flow evolution is provided. ( 2013 AIP Publishing LLC. [http://dx.doi.org/10.1063/1.4821361]

\section{INTRODUCTION}

The vortex breakdown (VB) development in one-fluid flows has been explored rather in detail. Early studies were reviewed by Escudier ${ }^{1}$ and more recent research is discussed in the monograph Counterflows. ${ }^{2}$ In contrast, VB in two-fluid flows has not attracted much attention. A few recent works include the experimental study, motivated by applications in bioreactors, ${ }^{3}$ and the numerical simulations of two-fluid flows in a sealed cylindrical container. ${ }^{4}$

An advantage of sealed cylinder is its closed domain with well-defined and controlled boundary conditions. This allows for meaningful comparisons of experimental and numerical results. They excellently agree for one-fluid VB flows as was first shown by Lopez. ${ }^{5}$

For free-surface flows however, some numerical and experimental results have discrepancies even in flow topology, not only in quantitative data. ${ }^{3,6}$ Brady et al.${ }^{4}$ provided a detailed review which indicates that further research is required of VB in free-surface and two-fluid flows.

Many works explored VB in a cylinder with one rotating end disk and fixed other walls. In contrast to one-fluid flows, the cylinder orientation with respect to gravity is substantial for two-fluid flows. Brady et al. ${ }^{4,7}$ considered the vertical cylinder with the bottom disk rotating. In this case, the interface deforms downward near the axis and upward near the sidewall, typical of whirlpools. Our paper considers the opposite orientation, where the top disk rotates. In this case, the interface deforms upward near the axis and downward near the sidewall, typical of water spouts. Another important difference is that we focus on air and water in contrast to rather exotic fluids in Refs. 4 and 7. The difference is huge in the light-to-heavy fluid density ratio, which is $\rho_{\mathrm{r}}=0.00122$ here and $\rho_{\mathrm{r}}=0.5284$ in Ref. 4.

A striking feature reported in our paper is the development of a thin circulation layer (TCL) in water adjacent to the entire interface. This layer, where the meridional motion circulates anticlockwise, separates bulk regions of clockwise circulation in air and water. Next, we show that TCL develops for the fluids studied in Refs. 4 and 7 as well. The TCL thickness decreases as the Reynolds number, Re, characterizing the rotation strength, increases.

Since for $\operatorname{Re}<3000$, one-fluid steady axisymmetric VB flows in a sealed cylinder are typically stable ${ }^{8}$ and our study is limited to $\operatorname{Re} \leq 2000$, we expect that the flows considered here are stable. This expectation, however, must be checked in further research.

In the rest of this paper, we formulate the problem in Sec. II, discuss the simulation results in Sec. III, and summarize the findings in Sec. IV. 


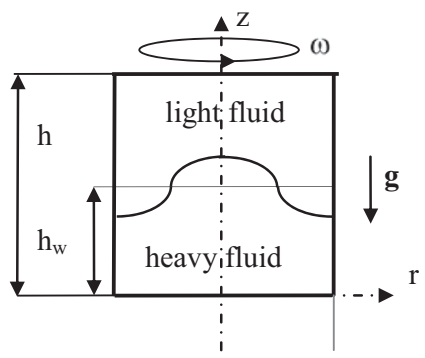

FIG. 1. Schematic of water-spout model.

\section{PROBLEM FORMULATION}

\section{A. Flow geometry}

Figure 1 is a schematic of the problem. The lower part, $0<\mathrm{z}<\mathrm{h}_{\mathrm{w}}$, of the cylindrical container is filled with a heavy fluid (e.g., water), the upper part, $\mathrm{h}_{\mathrm{w}}<\mathrm{z}<\mathrm{h}$, is filled with a light fluid (e.g., air); $\mathrm{h}$ is the cylinder height. With no motion, the interface is flat, $\mathrm{z}=\mathrm{h}_{\mathrm{w}}$ (thin horizontal line in Fig. 1). When the top disk $($ at $\mathrm{z}=\mathrm{h})$ rotates with angular velocity $\omega$, the interface becomes deformed as the curve in Fig. 1 schematically shows. This flow mimics a water spout where swirling air raises water near the rotation axis. This feature is opposite to that in a whirlpool where the water level decreases near the axis.

One control parameter is aspect ratio $\mathrm{H}=\mathrm{h} / \mathrm{R}$; $\mathrm{R}$ is the radius of the cylinder which serves as a length scale here. The dimensionless height of heavy fluid at rest is $H_{w}=h_{w} / R$. Other control parameters are the Reynolds number, $\mathrm{Re}=\omega \mathrm{R}^{2} / \nu_{\mathrm{a}}$, characterizing the swirl strength, the Froude number, $\mathrm{Fr}=\omega^{2} \mathrm{R} / \mathrm{g}$, which is a centrifugal-to-gravity acceleration ratio, and the Weber number, $\mathrm{We}=\rho_{\mathrm{w}} \omega^{2} \mathrm{R}^{3} / \sigma$, characterizing the effect of surface tension $\sigma$ at the interface; $\mathrm{g}=9.81 \mathrm{~m}^{2} / \mathrm{s}$ is the gravity acceleration. We first focus on the air-water flow and then consider the fluids studied by Brady et al. ${ }^{4,7}$ In the air-water case, $v_{\mathrm{a}}=15 \times 10^{-6} \mathrm{~m}^{2} / \mathrm{s}$ is the kinematic viscosity of air, $\rho_{\mathrm{w}}=1000 \mathrm{~kg} / \mathrm{m}^{3}$ is the water density, and $\sigma=0.0715 \mathrm{~kg} / \mathrm{s}^{2}$ at T $=300 \mathrm{~K}$. We assume that pressure on the interface at rest has its atmospheric value and the air density is $\rho_{\mathrm{a}}=1.22 \mathrm{~kg} / \mathrm{m}^{3}$.

Imagine a physical experiment where $\mathrm{R}=1 \mathrm{~mm}, a=v_{\mathrm{a}}{ }^{2} /\left(\mathrm{gR}^{3}\right)=0.0225$, and $b=\rho_{\mathrm{w}} v_{\mathrm{a}}{ }^{2} /(\mathrm{R} \sigma)$ $=0.00315$ are fixed, while $\omega$ eventually increases. Therefore, $\mathrm{Re}, \mathrm{Fr}=a \mathrm{Re}^{2}$, and $\mathrm{We}=b \mathrm{Re}^{2}$ also increase.

\section{B. Governing equations}

Using R, $\omega \mathrm{R}$, and $\rho_{\mathrm{w}} \omega^{2} \mathrm{R}^{2}$ as scales for length, velocity, and pressure, respectively, renders all variables dimensionless. We consider the steady axisymmetric flow of two viscous incompressible immiscible fluids governed by the Navier-Stokes equations, ${ }^{9}$

$$
\begin{aligned}
& \mathrm{r}^{-1} \partial(\mathrm{ru}) / \partial \mathrm{r}+\partial \mathrm{w} / \mathrm{z}=0, \\
& \mathrm{u} \partial \mathrm{u} / \partial \mathrm{r}+\mathrm{w} \partial \mathrm{u} / \partial \mathrm{z}=\mathrm{v}^{2} / \mathrm{r}-\rho_{\mathrm{n}} \partial \mathrm{p} / \partial \mathrm{r}+v_{\mathrm{n}} \operatorname{Re}^{-1}\left(\nabla^{2} \mathrm{u}-\mathrm{u} / \mathrm{r}^{2}\right), \\
& \mathrm{u} \partial \mathrm{v} / \partial \mathrm{r}+\mathrm{w} \partial \mathrm{v} / \partial \mathrm{z}+\mathrm{uv} / \mathrm{r}=v_{\mathrm{n}} \operatorname{Re}^{-1}\left(\nabla^{2} \mathrm{v}-\mathrm{v} / \mathrm{r}^{2}\right), \\
& \mathrm{u} \partial \mathrm{w} / \partial \mathrm{r}+\mathrm{w} \partial \mathrm{w} / \partial \mathrm{z}=-\rho_{\mathrm{n}} \partial \mathrm{p} / \partial \mathrm{z}+v_{\mathrm{n}} \operatorname{Re}^{-1}\left(\nabla^{2} \mathrm{w}\right),
\end{aligned}
$$

where $\nabla^{2} \equiv \mathrm{r}^{-1} \partial(\mathrm{r} \partial / \partial \mathrm{r}) / \partial \mathrm{r}+\partial^{2} / \partial \mathrm{z}^{2},(\mathrm{u}, \mathrm{v}, \mathrm{w})$ are the velocity components in the cylindrical coordinate $(\mathrm{r}, \phi, \mathrm{z})$, and $\mathrm{p}$ is pressure. The coefficients, $\rho_{\mathrm{n}}$ and $v_{\mathrm{n}}$, are both equal 1 at $\mathrm{n}=1$ (in the heavy fluid) while $\rho_{\mathrm{n}}=\rho_{\mathrm{w}} / \rho_{\mathrm{a}}$ and $v_{\mathrm{n}}=v_{\mathrm{a}} / v_{\mathrm{w}}$ at $\mathrm{n}=2$ (in the light fluid). For the air-water case, $v_{\mathrm{w}}=10^{-6}$ $\mathrm{m}^{2} / \mathrm{s}$ is the kinematic viscosity of water. 


\section{Boundary conditions}

Equations (1)-(4) are solved under the following boundary conditions:

(i) Regularity at the axis, $0<\mathrm{z}<\mathrm{H}, \mathrm{r}=0$ : $\mathrm{u}=\mathrm{v}=0, \partial \mathrm{w} / \partial \mathrm{r}=0$.

(ii) No-slip at the walls: $\mathrm{u}=\mathrm{v}=\mathrm{w}=0$ at the still disk, $0<\mathrm{r}<1, \mathrm{z}=0$, and at the sidewall, $0<\mathrm{z}<\mathrm{H}, \mathrm{r}=1 ; \mathrm{u}=\mathrm{w}=0, \mathrm{v}=\mathrm{r}$ at the rotating disk, $0<\mathrm{r}<1, \mathrm{z}=\mathrm{H}$.

(iii) Continuity of all the velocity and stress components at the interface, $z=\mathrm{F}(\mathrm{r})$. In particular, the balance for the normal stresses yields that

$$
\mathrm{p}_{\mathrm{w}}-\mathrm{p}_{\mathrm{a}}=\mathrm{We}^{-1} \nabla \cdot \mathbf{n}-\mathrm{Re}^{-1} \mathbf{n} \cdot\left(\boldsymbol{\tau}_{\mathrm{w}}-\mu_{\mathrm{r}} \boldsymbol{\tau}_{\mathrm{a}}\right) \cdot \mathbf{n}-\mathrm{Fr}^{-1}\left(1-\rho_{\mathrm{r}}\right) \mathrm{z}+\mathrm{C},
$$

where $\mathbf{n}$ is the unit vector normal to the interface, $\boldsymbol{\tau}_{\mathrm{w}}$ and $\boldsymbol{\tau}_{\mathrm{a}}$ are tensors of the viscous stresses in the heavy and light fluids, respectively, $\mu_{\mathrm{r}}$ and $\rho_{\mathrm{r}}$ are the light-to-heavy fluid ratios of the dynamic viscosities and densities, respectively, and $\mathrm{C}$ is a constant which is determined by imposing the mass conservation of the heavy fluid inside the container as the interface is deformed,

$$
2 \int_{0} \int^{1} \mathrm{~F}(\mathrm{r}) \mathrm{rdr}=\mathrm{H}_{\mathrm{w}} .
$$

The conditions at the interface are similar to those formulated by Brady et al. ${ }^{4,7}$

The numerical procedure, used in this paper, is described in detail in our previous study ${ }^{10}$ where comparison with the results by Brady et al. ${ }^{4}$ is made, which validates the code.

\section{DEVELOPMENT OF THIN LAYER WITH ANTICLOCKWISE MERIDIONAL CIRCULATION}

\section{A. Vortex breakdown near the bottom disk center}

First, we consider the air-water flow at $\mathrm{H}=1$ and equal volumes of air and water, $\mathrm{H}_{\mathrm{w}}=0.5$. The flow topology is illustrated by streamlines of the meridional motion, which are contours $\Psi=$ constant, where $\Psi$ is the Stokes stream function, $\mathrm{u}=\partial \Psi / \partial \mathrm{z}, \mathrm{w}=-\partial \Psi / \partial \mathrm{r}$. Figure 2 shows streamline patterns at $\operatorname{Re}=267$ (a) and $\operatorname{Re}=300$ (b). The rotating top dick generates the centrifugal force, which pushes air to periphery near the disk similar to that in the Kárman boundary layer. ${ }^{11}$

The Kárman pumping drives the clockwise circulation of air in region CR1 (Fig. 2(a)). This flow induces the anticlockwise circulation of water in region CR2, which in turn generates the Moffat corner eddies. ${ }^{12}$ Among them, only the largest eddy is resolved by our standard grid and denoted as ME in the lower-right corner of the water flow in Fig. 2.

The flow pattern, presented in Fig. 2(a), remains topologically invariant as Re diminishes to zero. In contrast as Re increases, the clockwise meridional circulation (vortex breakdown, VB) develops near the axis-bottom intersection point in region CR3 (Fig. 2(b)). Figure 3 depicts the
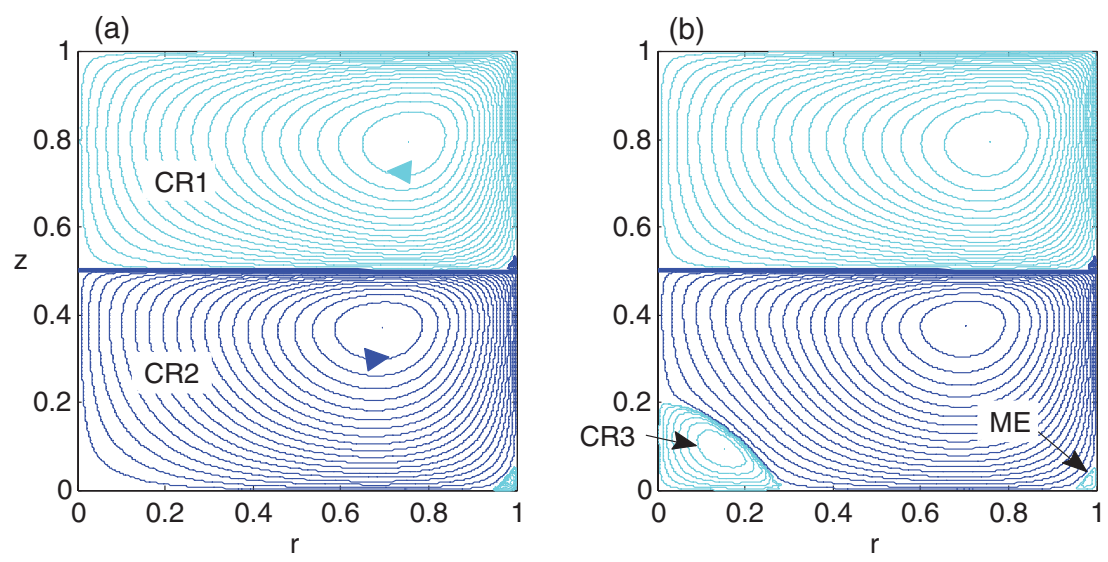

FIG. 2. Streamline patterns at $\operatorname{Re}=267$ (a) and $\mathrm{Re}=300$ (b) show development of circulation region CR3. 


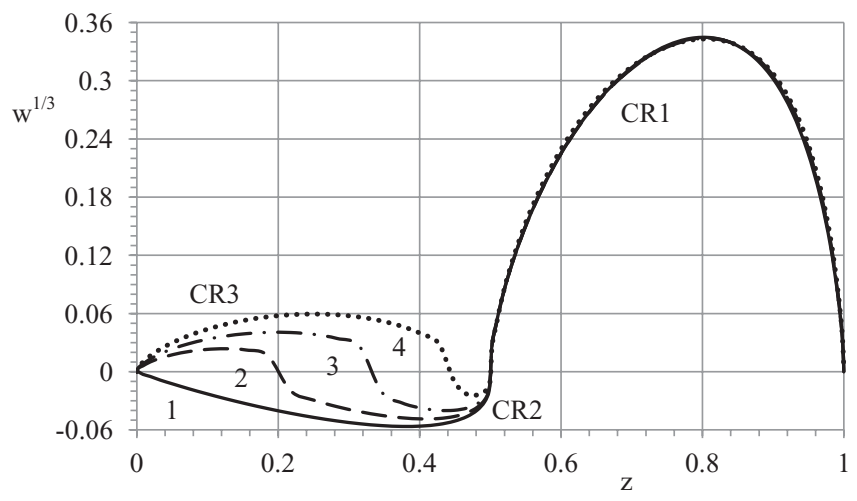

FIG. 3. Profiles of velocity $\mathrm{w}$ on the axis at $\operatorname{Re}=267$ (1), $\operatorname{Re}=300$ (2), $\operatorname{Re}=367$ (3), and $\operatorname{Re}=400$ (4) show the emergence and expansion of region CR3 (see Fig. 2(b)).

distribution of velocity $w$ on the axis, $r=0$, at $\operatorname{Re}=267$ (curve 1 ), $\operatorname{Re}=300$ (curve 2 ), $\operatorname{Re}=367$ (curve 3), and $\operatorname{Re}=400$ (curve 4). We plot $\mathrm{w}^{1 / 3}(\mathrm{z}$ ) in Fig. 3 for convenient observation of regions in water where $\mathrm{w}$ is very small compared with $\mathrm{w}$ values in air. At $\mathrm{Re}=267$ (curve 1 in Fig. 3), $\mathrm{w}$ is positive in air and negative in water. The w profile in air remains nearly unchanged in this range of Re. In contrast, $\mathrm{w}(\mathrm{z})$ significantly varies in water. Interpolation yields that region CR3, where $\mathrm{w}>0$ in water, emerges at $R e=R_{1}=270$. As Re increases, the $w>0$ region (CR3) expands and the $\mathrm{w}<0$ region (CR2) shrinks in water as curves 2, 3, and 4 illustrate in Fig. 3.

The water motion is driven by two factors: (i) meridional and (ii) swirl shear stresses at the interface, both provided by the air flow. For small Re, factor (i) dominates and develops the anticlockwise circulation in region CR2 (Fig. 2(a)). The effect of swirl on the meridional motion of water occurs via the centrifugal force, $\mathrm{v}^{2} / \mathrm{r}$. Since it is proportional to the squared swirl velocity, its effect is negligible compared with that of the meridional stresses in a slow flow.

The meridional motion in region CR2 (Fig. 2(a)), being directed upward near the sidewall, blocks the transfer of angular momentum downward and transports it toward the axis near the interface and then toward the bottom disk near the axis. Therefore as Re increases, swirl mostly affects the water flow near the axis-bottom intersection and results in the development of counter-circulation region CR3 (Fig. 2(b)).

Physical reasoning behind the region CR3 development is similar to that for the Bödewadt boundary layer. ${ }^{13}$ If a rotating fluid meets a normal wall, a secondary flow develops, which converges to the rotation axis near the wall and goes away from the wall near the axis. The centrifugal force balances the radial gradient of pressure away from the wall, $\partial \mathrm{p} / \partial \mathrm{r}=\mathrm{v}^{2} / \mathrm{r}$, but has the second-order zero at the wall due to the no-slip condition, $v=0$. The pressure gradient, being unbalanced by the centrifugal force, pushes the fluid to converge near the wall (Bödewadt pumping) that results in a tornado-like outflow near the rotation axis.

For small Re, the Bödewadt pumping is too weak to reverse the CR2 circulation. As the rotation speeds up, i.e., Re increases, the tornado-like motion intensifies and overcomes the CR2 circulation in the axis-bottom vicinity (Fig. 2(b)). The circulation reversal occurs as $\operatorname{Re}$ exceeds $\operatorname{Re}_{1}$. The reversal results in that region CR3 emerges and eventually expands for larger Re; compare Figs. 2(b) and 4(a).

\section{B. Merging of vortex breakdown bubble and corner eddy}

In contrast to CR3, region CR2 shrinks as Re increases. Curves 2, 3, and 4 in Fig. 3 illustrate this trend showing how the distribution of velocity on the axis varies. Figures 2(b) and 4(a) also illustrate the CR3 radial expansion.

At $R e=R_{2}$, which is around 580, vortex breakdown region CR3 and Moffatt corner eddy ME merge. The two saddle stagnation points of meridional motion ( $S_{1}$ and $S_{2}$ in Fig. 4(a)) coalesce and 

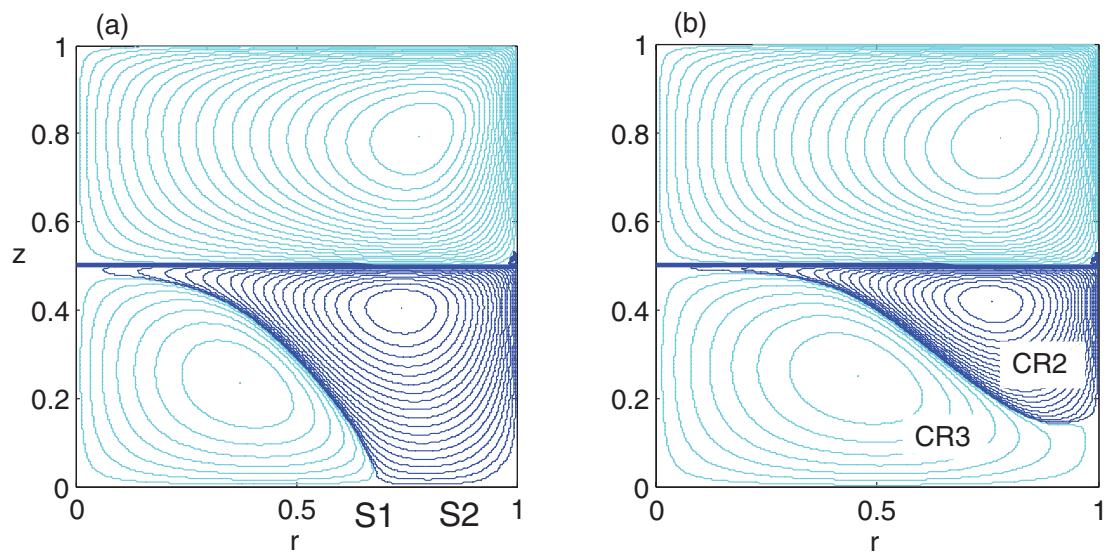

FIG. 4. Streamline patterns at $\operatorname{Re}=467$ (a) and $\operatorname{Re}=600$ (b) show separation of region CR2 from bottom.

disappear as Re increases. As a result, circulation region CR2 becomes separated from the bottom. Figure 5 is a schematic helping to explain the separation process details.

The bold lines represent the bottom disk and the sidewall, thin curves are streamlines, the arrows indicate the flow directions, and the half-bold curves separate the opposite-circulation regions. Saddle points $\mathrm{S} 1$ and $\mathrm{S} 2$ in Fig. 4(a) merge into saddle point $\mathrm{S}$ at $\mathrm{Re}=\operatorname{Re}_{2}$ (Fig. 5(a)), which separates from the bottom disk for $\operatorname{Re}>\operatorname{Re}_{2}$ (Fig. 5(b)). As Re further increases, saddle $S$ and the center, depicted by the bold point in Fig. 5(b), coalesce at some $R e=R_{3}$, which is slightly larger than $\operatorname{Re}_{2}$, and disappear for $\operatorname{Re}>\operatorname{Re}_{3}$ (Fig. 4(b)). This bifurcation scenario was systematically studied by Brøns. ${ }^{14}$ Figure 5 also depicts the smaller Moffatt eddy at the right-lower corner (not resolved in Fig. 4). The merging of CR3 and ME develops the vortex breakdown region (again denoted as CR3), which extends from the axis to the sidewall for $\operatorname{Re}>\operatorname{Re}_{2}$ (Fig. 4(b)).

Figure 6 depicts profiles of swirl, $v$, and meridional, $v_{t}$, velocities at the interface for $\operatorname{Re}=600$; subscript " $\mathrm{t}$ " underlines that $\mathrm{v}_{\mathrm{t}}$ is tangent to the interface (and normal to the swirl velocity). The velocity distribution at the interface drives the water flow and, therefore, helps explain the pattern shown in Fig. 4(b). Since $v$ is significantly larger than $\left|v_{t}\right|$ (Fig. 6), region CR3, where the meridional motion is driven by the centrifugal force, is large compared with region CR2, where the meridional circulation is driven by $\mathrm{v}_{\mathrm{t}}$. The $\left|\mathrm{v}_{\mathrm{t}}\right| \mathrm{v}$ ratio is very small near the axis and, accordingly, region CR2 is very thin there (Fig. 4(b)).

\section{Transformation of region CR2 into thin layer}

The dominance of swirl velocity over the meridional velocity at the interface becomes more prominent, as Re increases. Figure 7 illustrates this by depicting the dependence on Re of the maximal values of swirl, $\mathrm{v}_{\mathrm{m}}$, and meridional, $\mathrm{v}_{\mathrm{tm}}$, velocity magnitudes at the interface. In the entire range of $\mathrm{Re}, \mathrm{v}_{\mathrm{m}}$ is larger than $\mathrm{v}_{\mathrm{tm}}$. For small $\mathrm{Re}, \mathrm{v}_{\mathrm{tm}}$ increases proportionally to Re due to the Kárman pumping. However, after region CR3 emerges in water, $v_{t m}$ reaches its maximal value

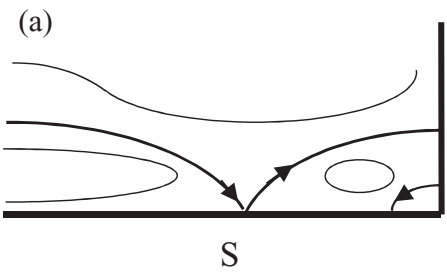

(b)

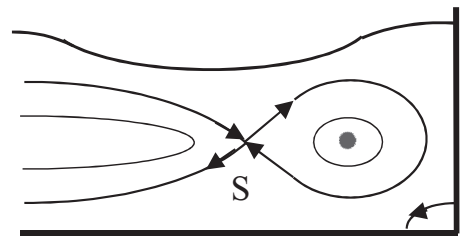

FIG. 5. Schematic shows (a) merging of saddle points S1 and S2 in Fig. 4(a) into saddle point S and (b) separation of S from the bottom disk. Arrows indicate flow direction. 


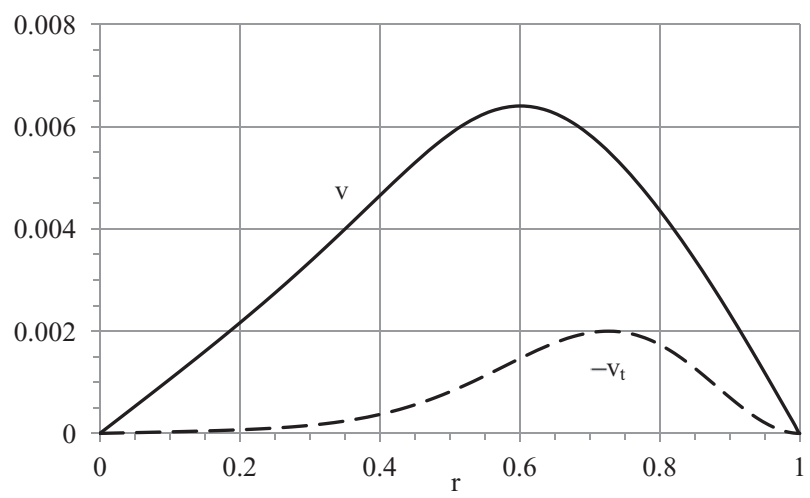

FIG. 6. Distribution of swirl, v, and meridional, $\mathrm{v}_{\mathrm{t}}$, velocity on interface at $\mathrm{Re}=600$.

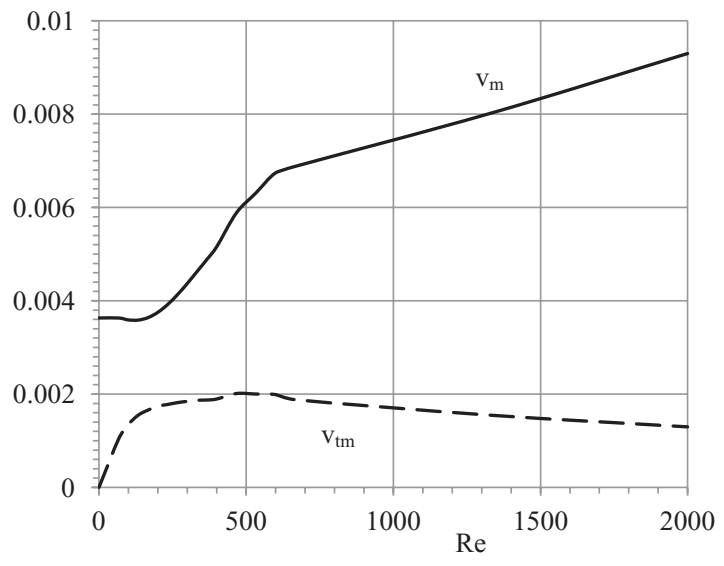

FIG. 7. Dependence of maximal swirl, $\mathrm{v}_{\mathrm{m}}$, and meridional, $\mathrm{v}_{\mathrm{tm}}$, velocity magnitudes at the interface on Reynolds number.

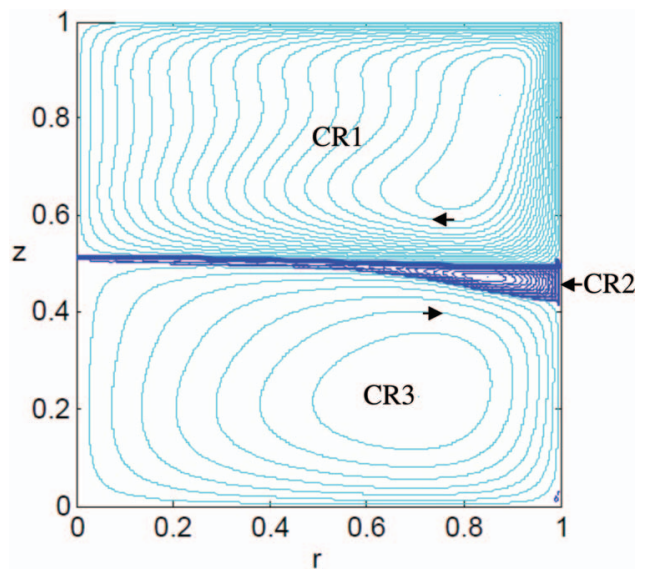

FIG. 8. Streamline pattern at $\mathrm{Re}=2000$ shows thin region of anticlockwise circulation in water, separating regions of clockwise meridional circulation. 


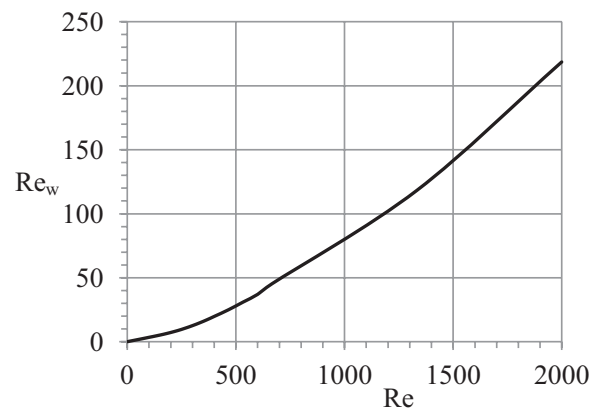

FIG. 9. Dependence between the Reynolds numbers, characterizing the water $\left(\operatorname{Re}_{\mathrm{w}}\right)$ and air $(\operatorname{Re})$ flows.

and then decreases. The decrease occurs because the Bödewadt pumping, generating the clockwise meridional motion in region $\mathrm{CR} 3$, suppresses the anticlockwise motion in region CR2. In contrast to decreasing $\mathrm{v}_{\mathrm{tm}}, \mathrm{v}_{\mathrm{m}}$ linearly grows for large Re.

This effect makes even larger the $\mathrm{v}_{\mathrm{m}} / \mathrm{v}_{\mathrm{tm}}$ ratio, i.e., the dominance of the Bödewadt pumping over the $v_{t}$ effect. This positive feedback yields that the thickness of region CR2 diminishes. First, this mechanism makes region CR2 thin near the axis (Fig. 4(b)). As Re increase, the CR2 thickness becomes small everywhere, including the sidewall vicinity, as Fig. 8 illustrates at $\operatorname{Re}=2000$. However, region CR2 cannot totally disappear, because $\mathrm{v}_{\mathrm{tm}}>0$ (Fig. 7) and regions of the clockwise meridional circulation, CR1 in air and CR3 in water, have opposite velocity directions near the interface and therefore must be separated by a region of the anticlockwise circulation (CR2).

(a)

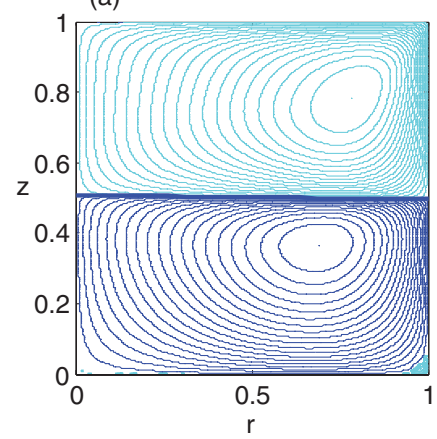

(d)

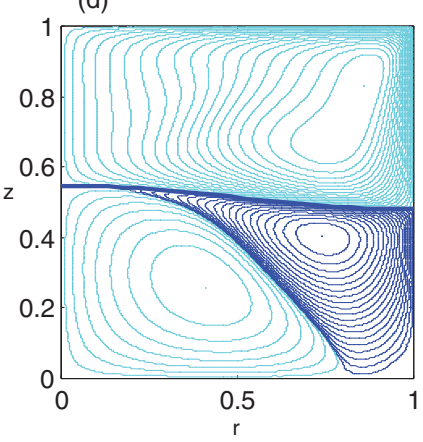

(b)

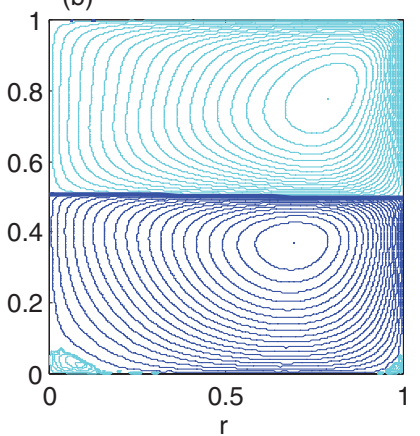

(e)

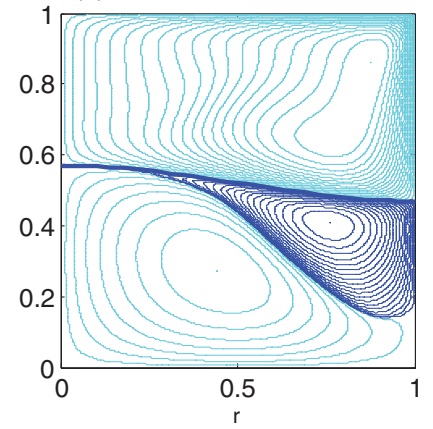

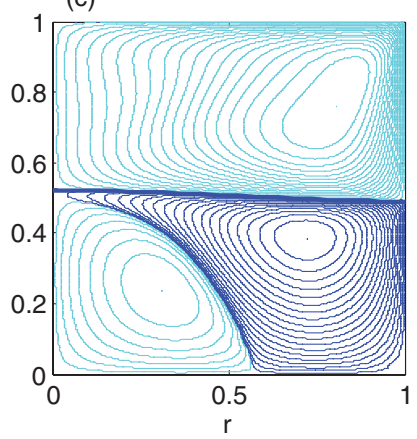

(f)

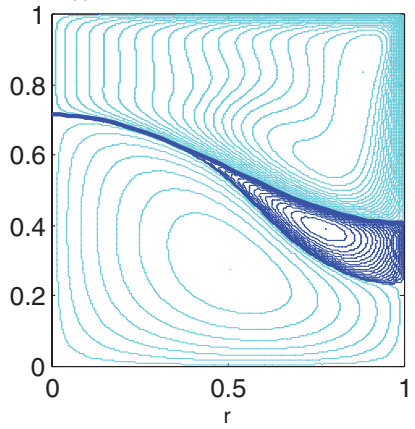

FIG. 10. Development of thin separation layer in the flow of two fluids with close densities: (a) $\operatorname{Re}=200$, (b) $\operatorname{Re}=220$, (c) $\operatorname{Re}=350$, (d) $\operatorname{Re}=500$, (e) $\operatorname{Re}=600$, and (f) $\operatorname{Re}=1000$. 


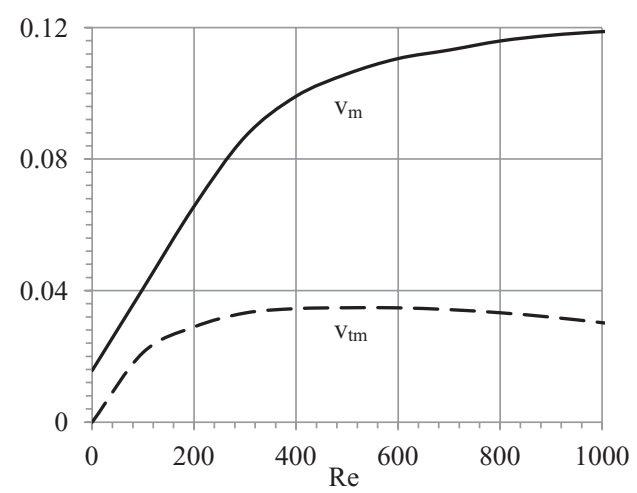

FIG. 11. Dependence of maximal swirl, $\mathrm{v}_{\mathrm{m}}$, and meridional, $\mathrm{v}_{\mathrm{tm}}$, velocity magnitudes at interface on Reynolds number in flow of two fluids with close densities.

Since the maximal swirl velocity in air (which equals 1) is significantly larger than that in water (Fig. 7), the air flow is more pressed by the centrifugal force toward the sidewall than the water flow is. Accordingly the center of meridional circulation in air is located closer to the sidewall than that in water in Fig. 8.

Since the Bödewadt pumping dominates over the $\mathrm{v}_{\mathrm{t}}$ effect for large Re, the strength of water flow can be characterized by the Reynolds number, $\operatorname{Re}_{\mathrm{w}}=(\mathrm{rv})_{\max } / v_{\mathrm{w}}$, where $(\mathrm{rv})_{\max }$ is the maximal value of angular momentum rv, which is achieved at the interface. Figure 9 depicts the dependence of $\mathrm{Re}_{\mathrm{w}}$, characterizing the water motion, on $\mathrm{Re}$, characterizing the air motion.

\section{Development of thin separation layer in flow of two fluids with close densities}

The interface deformation in the air-water flow is small even at $\operatorname{Re}=2000$ (Fig. 8), where the maximal (minimal) height of interface is $0.51(0.49)$. The deformation is small because the water density is about thousand times the air density. To explore, whether the TCL development occurs for other fluids as well, and to observe large deformations of interface in a steady axisymmetric flow, we address now the media used in Ref. 4, where the light-to-heavy fluid density ratio is $\rho_{\mathrm{r}}=0.5284$ and dynamic viscosity ratio $\mu_{\mathrm{r}}=0.2$. We keep the same values of the aspect ratio $(\mathrm{H}=1)$ and the height of the heavy fluid at rest $\left(\mathrm{H}_{\mathrm{h}}=0.5\right)$.

Figure 10 depicts streamlines of the meridional motion at Re values shown near the pictures. Here $\mathrm{Re}=\omega \mathrm{R}^{2} / \nu_{\mathrm{h}}$ where $v_{\mathrm{h}}$ is the kinematic viscosity of the heavy (lower) fluid. The flow topology transformations and the development of the thin separation layer, illustrated by Fig. 10, are similar to those in the air-water flow. The difference is in the significant deformation of interface as Fig. 10(f) depicts at $\operatorname{Re}=1000$. Figure 11 is an analog of Fig. 7 and reveals the same features: (i) dominance of maximal swirl velocity, $\mathrm{v}_{\mathrm{m}}$, over the maximal meridional velocity magnitude, $\mathrm{v}_{\mathrm{tm}}$, on the interface and (ii) decreasing of $\mathrm{v}_{\mathrm{tm}}$ for large Re. Thus, the flow features revealed for the air-water case remains similar for the media used in Ref. 4.

\section{CONCLUSION}

The numerical simulations of steady axisymmetric flows of two viscous incompressible immiscible fluids in a sealed vertical container with the rotating top disk reveal the development of counter-circulation (vortex breakdown, VB) region of the meridional motion near the bottom center, as the rotation speeds up (Fig. 2). If the rotation further increases, the VB region with the clockwise meridional circulation expands and occupies nearly the entire water domain, being detached from the interface by a thin separation layer of the anticlockwise meridional circulation (Fig. 8). The TCL development is common for the air-water flow and for the flow of the two fluids studied in Ref. 4 (Fig. 10). This indicates that the flow pattern, consisting of two global regions, where the meridional circulation is clockwise, and the thin separating layer of anticlockwise circulation, is typical of 
water-spout-like high-speed two-fluid flows. Physical reasoning behind this pattern development is provided in terms of the Bödewadt and Kárman pumping mechanisms.

\section{ACKNOWLEDGMENTS}

Partial support from the Ministry of Science and Education and Junta de Andalucía (Spain) through Grant Nos. DPI2010-21103 and TEP-7465, respectively, is gratefully acknowledged.

${ }^{1}$ M. P. Escudier, "Vortex breakdown: observations and explanations," Prog. Aerosp. Sci. 25, 189 (1988).

${ }^{2}$ V. N. Shtern, Counterflows (Cambridge University Press, New York, 2012).

${ }^{3}$ D. Lo Jacono, M. Nazarinia, and M. Brøns, "Experimental vortex breakdown topology in a cylinder with a free surface," Phys. Fluids 21, 111704 (2009).

${ }^{4}$ P. T. Brady, M. Herman, and J. M. Lopez, "Two-fluid confined flow in a cylinder driven by a rotating end wall," Phys. Rev. E 85, 016306 (2012)

${ }^{5}$ J. M. Lopez, “Axisymmetric vortex breakdown Part 1. Confined swirling flow,” J. Fluid Mech. 221, 533 (1990).

${ }^{6} \mathrm{M}$. Brøns, L. K. Voigt, and J. N. Sørensen, "Topology of vortex breakdown bubbles in a cylinder with rotating bottom and free surface," J. Fluid Mech. 428, 133 (2001).

${ }^{7}$ P. T. Brady, M. Herman, and J. M. Lopez, "Addendum to "Two-fluid confined flow in a cylinder driven by a rotating end wall," Phys. Rev. E 85, 067301 (2012).

${ }^{8}$ A. Yu. Gelfgat, P. Z. Bar-Yoseph, and A. Solan, "Three-dimensional instability of axisymmetric flow in a rotating lid cylinder enclosure,” J. Fluid Mech. 438, 363-377 (2001).

${ }^{9}$ G. K. Batchelor, An Introduction to Fluid Dynamics (Cambridge University Press, Cambridge, 1967).

${ }^{10}$ M. A. Herrada, V. N. Shtern, and J. M. Lopez-Herrera, "Off-axis vortex breakdown in a shallow whirlpool," Phys. Rev. E 87, $063016(2013)$.

${ }^{11}$ T. von Kárman, "Laminare und turbulente Reibang,” Z. Angew. Math. Mech. 1, 233 (1921).

${ }^{12}$ H. K. Moffatt, "Viscous and resistive eddies near a sharp corner," J. Fluid Mech. 18, 1 (1964).

${ }^{13}$ U. T. Bödewadt, "Die Drehströmung über festem Grund," Z. Angew. Math. Mech. 20, 241 (1940).

${ }^{14}$ M. Brøns, "Streamline topology: Patterns in fluid flows and their bifurcations," Adv. Appl. Mech. 41, 1-42 (2007). 